\title{
DIÂMETRO DE TRADO NECESSÁRIO À COLETA DE AMOSTRAS NUM CAMBISSOLO SOB PLANTIO DIRETO OU SOB PLANTIO CONVENCIONAL ANTES OU DEPOIS DA ARAÇÃO ${ }^{(1)}$
}

\author{
André Guarçoni M. ${ }^{(2)}$, Víctor Hugo Alvarez V. ${ }^{(3)}$, Roberto Ferreira \\ Novais ${ }^{(3)}$, Reinaldo Bertola Cantarutti ${ }^{(3)}$, Helio Garcia Leite ${ }^{(4)} \&$ \\ Francisco Morel Freire ${ }^{(5)}$
}

\begin{abstract}
RESUMO
Quando se recomenda um número definido de amostras simples de solo para formar uma amostra composta, não se leva em consideração o diâmetro do trado a ser utilizado na amostragem. Visando a determinação do diâmetro de trado que permitisse redução no esforço e no tempo necessários à amostragem, realizou-se um estudo em que foram coletadas amostras simples de solo com seis volumes diferentes $\left(40,90,160,360,810\right.$ e $\left.1.000 \mathrm{~cm}^{3}\right)$, em três situações de preparo-coleta (plantio direto (PD) ou plantio convencional antes (PCAA) ou depois da aração (PCDA)), no intuito de se obter o volume de amostra simples com o qual se estimasse

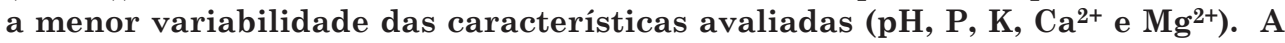
estimativa da variabilidade da maioria das características químicas da fertilidade do solo avaliadas foi, de maneira geral, semelhante entre o plantio direto (PD) e o plantio convencional antes da aração (PCAA), sendo maior em ambos os casos do que no plantio convencional depois da aração (PCDA). O aumento do volume das amostras simples, para uma mesma profundidade de coleta, reduziu a estimativa da variabilidade das características químicas da fertilidade avaliadas até valores
\end{abstract}

\footnotetext{
(1) Parte da Tese de Doutorado do primeiro autor. Recebido para publicação em junho de 2006 e aprovado em maio de 2007.

${ }^{(2)}$ Pesquisador do Instituto Capixaba de Pesquisa. Assistência Técnica e Extensão Rural - INCAPER. CRDR-CS, BR 262, Km 94, CEP 29375-000 Venda Nova do Imigrande (ES). E-mail: guarconi@incaper.es.gov.br

${ }^{(3)}$ Professor do Departamento de Solos, Universidade Federal de Viçosa - UFV. CEP 36571-000 Viçosa (MG). E-mails: vhav@ufv.br; rfnovais@ufv.br; cantarutti@ufv.br

(4) Professor do Departamento de Engenharia Florestal, UFV. E-mail: hgleite@ufv.br

(5) Pesquisador da EPAMIG-MG. Centro Tecnológico do Centro-Oeste - CTCO. Rod. MG 424, Km 64, CEP $35701-970$ Sete Lagoas (MG). E-mail: francisco.morel@epamig.br
} 
que praticamente se estabilizaram. Isso permitiu a recomendação de um trado com diâmetro de aproximadamente $5,4 \mathrm{~cm}$, a ser utilizado para coleta de $\mathrm{n}$ amostras simples que apresentem definida variação tolerada em torno da média (f) na obtenção de resultados analíticos, sendo 20 amostras simples no plantio direto $(f=$ $20 \%), 15$ no plantio convencional antes da aração $(f=20 \%)$ e 10 no plantio convencional depois da aração ( $(=10 \%)$.

Termos de indexação: amostragem, amostras simples, variabilidade superficial.

\title{
SUMMARY: AUGER DIAMETER FOR SAMPLING AN INCEPTISOL UNDER NO-TILL OR CONVENTIONAL PLANTING, BEFORE OR AFTER TILLAGE
}

\begin{abstract}
When a determined number of single soil samples is recommended for the formation of a composite sample, the diameter of the sampling auger is not taken into account. Thus, this study aimed to determine the auger diameter that would allow a reduction in the time and effort needed for soil sampling. Single soil samples of six different volumes (40, 90, 160, 360, 810 and 1,000 $\mathrm{cm}^{3}$ ) were collected in three management-harvest systems (no-till planting $(P D)$, conventional planting before (PCAA) or after tillage (PCDA), in order to obtain the single sample volume to estimate the lowest variability of the evaluated characteristics ( $\mathrm{pH}, \mathrm{P}, \mathrm{K}, \mathrm{Ca}^{2+}$ and $\mathrm{Mg}^{2+}$ ). In general, the estimate of the variability for most chemical characteristics of soil fertility was similar under no-till planting $(P D)$ and conventional planting before tillage (PCAA), and higher than that under conventional planting after tillage (PCDA); the increase in volume of the single samples at a same collection depth reduced the variability estimate of the soil fertility characteristics until practically stable values. This allowed the recommendation of an auger diameter of approximately $5.4 \mathrm{~cm}$ for the collection of $n$ single samples, i.e., 20 single samples for the soils under no-till ( $f=20 \%), 15$ in those under conventional planting before tillage $(f=$ $20 \%$ ) and 10 in those under conventional planting after tillage ( $f=10 \%)$. Such values ensure tolerable definite variation around the average (f) to establish the analytical results.
\end{abstract}

Index terms: sampling, single samples, surface variability.

\section{INTRODUÇÃO}

Muitos erros potenciais estão envolvidos num programa de adubação em razão da elevada variabilidade de características químicas, um dos mais importantes é gerado pela amostragem de solo inadequada (Mallarino, 1996). Na avaliação da fertilidade média do solo, a amostra que comumente se utiliza para representar a área a ser corrigida e, ou, adubada é a "amostra composta". Cada amostra composta é formada pela mistura homogênea de um número predefinido de "amostras simples" de pequeno volume (Cline, 1944).

Segundo Upchurch \& Edmonds (1991), há três fatores distintos envolvidos na amostragem de solo para uma característica particular: número de amostras a ser coletado, local de coleta e volume da amostra. Acrescentando outros fatores além dos citados, Schlindwein \& Anghinoni (2002) afirmam que o número e a distribuição das amostras simples devem contemplar as macrovariações (>2 m); já a forma, o volume e o local de amostragem devem contemplar as meso $(0,05$ a $2 \mathrm{~m})$ e as microvariações $(<5 \mathrm{~cm})$. Entretanto, na determinação do número de amostras simples, necessário para formar uma amostra composta representativa, a estimativa da variabilidade de determinada característica do solo é considerada como um todo, e as meso e microvariações apresentam grande influência nessa estimativa. Dessa forma, a estimativa da variabilidade pode ser aumentada ou diminuída, de acordo com o grau de influência de cada um dos fatores envolvidos na amostragem de solo, uma vez que estes estão intrinsecamente relacionados.

É de consenso na literatura que, ao se aumentar o tamanho da amostra (número de amostras simples), a estimativa da variabilidade vai diminuindo até atingir um valor mínimo, a partir do qual os decréscimos nessa estimativa são insignificantes em relação ao aumento do número de amostras simples (McBratney \& Webster, 1983; Webster \& Burgess, 1984; Burrough, 1991).

Esse fato pode ser extrapolado para o volume das "amostras simples", ou seja, quanto maior o volume de solo coletado em cada "amostra simples", menor a 
estimativa da variabilidade de determinada característica avaliada, como observado por Schlindwein \& Anghinoni (2002) e Alvarez V. \& Guarçoni M. (2003), para características químicas do solo; por Fante Jr. et al. (1999), para distribuição do sistema radicular de aveia forrageira; e por Silva \& Costa (1998), para larvas de Coleoptera em plantio direto. No entanto, os autores citados não determinaram modelos que explicassem a variabilidade em função do volume das amostras, uma vez que não utilizaram o artifício estatístico proposto no presente trabalho, ou seja, não consideraram grupos de amostras como repetições, o que permitiria estimar o erro entre as estimativas das variabilidades obtidas em cada volume de amostra estudado.

Em se tratando de características químicas do solo, ao se aumentar o volume das amostras simples, para uma mesma profundidade de amostragem, as microvariações $(<5 \mathrm{~cm})$ são sucessivamente incorporadas ao volume de solo coletado, diminuindo a estimativa da variabilidade, o que reduz o número de amostras simples necessário para formar uma amostra composta representativa de um talhão homogêneo. Na prática, essa redução deve levar em conta a redução do esforço e, ou, do tempo necessários para se realizar a amostragem, de forma que o número de amostras simples e o volume de solo a ser coletado propiciem uma amostragem rápida e com o menor esforço possível.

A maioria das características químicas do solo apresenta maior variabilidade no sistema plantio direto do que no plantio convencional (araçãogradagem), devido à manutenção das linhas de adubação no primeiro caso (Salet et al., 1996; Anghinoni \& Salet, 1998; Oliveira et al., 2002). No plantio convencional, pela mesma razão, a variabilidade é maior antes da aração-gradagem do que após o preparo (Santos \& Vasconcelos, 1987; Guarçoni M. et al., 2006). Contudo, o aumento do volume da amostra simples pode não reduzir a variabilidade das características avaliadas na mesma magnitude, considerando essas três situações.

Este trabalho teve por objetivos: estudar a influência do volume da amostra simples na determinação da variabilidade de características químicas da fertilidade do solo e calcular o diâmetro de trado necessário para coleta de determinado número de amostras simples, em um Cambissolo sob plantio direto ou sob plantio convencional antes ou depois da aração.

\section{MATERIAL E MÉTODOS}

Foram coletadas amostras simples em um Cambissolo Háplico argiloso (área experimental da Agronomia - UFV) (14, 8, 22 e 56 \% de areia grossa, areia fina, silte e argila, respectivamente) sob dois tipos de preparo: plantio direto (PD) e plantio convencional (aração com arado de discos até $20 \mathrm{~cm}$ de profundidade e duas passadas de grade niveladora). No plantio convencional, foram utilizados dois momentos de coleta: antes da aração-gradagens (PCAA) e depois da aração-gradagens (PCDA). Dessa forma, foram individualizadas três situações de preparo-coleta (PD, PCAA e PCDA).

No PD, sucessão milho-feijão (out-mar: milho, marjul: feijão e jul-out: pousio), que apresentava sete anos desde a implantação, as amostras simples de solo foram coletadas no momento em que estava sendo cultivado feijão no espaçamento de 0,45 m entre linhas. Já no PCAA, as amostras simples de solo foram coletadas logo após a colheita de milho, cultivado no espaçamento de 0,90 m entre linhas, em uma área onde se cultivava milho havia alguns anos e estava localizada a, aproximadamente, $50 \mathrm{~m}$ da área do PD. O PCDA estava localizado em uma área distanciada de, aproximadamente, $20 \mathrm{~m}$ da área do PCAA, onde há dois anos havia sido cultivado algodão, permanecendo o solo em pousio nesse intervalo de dois anos.

Em cada uma das três situações de preparo-coleta, foram coletadas amostras simples de solo com seis volumes diferentes $\left(40,90,160,360,810\right.$ e $\left.1.000 \mathrm{~cm}^{3}\right)$, correspondendo, respectivamente, a prismas de base quadrada, medindo $2 \times 2,3 \times 3,4 \times 4,6 \times 6,9 \times 9$ e 10 x $10 \mathrm{~cm}$ de lado por $10 \mathrm{~cm}$ de profundidade. Foram coletadas três amostras para cada volume estudado, sendo, cada uma, formada por 10 amostras simples (10 prismas). Com isso, foram obtidas 18 amostras por situação de preparo-coleta, formadas por um total de 180 amostras simples (180 prismas).

Para coleta das amostras simples de solo utilizaram-se lâminas galvanizadas com $1 \mathrm{~mm}$ de espessura x $10 \mathrm{~cm}$ de altura $\mathrm{x}$ largura correspondente à seção $(2,3,4,6,9$ ou $10 \mathrm{~cm})$.

Para o PD e para o PCAA, as amostras simples foram coletadas da seguinte maneira: nos volumes de 40,90 e $160 \mathrm{~cm}^{3}, 30$ amostras simples (30 prismas) foram coletadas uma ao lado da outra, perpendicularmente ao sulco, sendo 15 de cada lado do meio do sulco (Figura 1). Nos volumes de 360, 810 e $1.000 \mathrm{~cm}^{3}$, foram coletadas 10 amostras simples, uma ao lado da outra, perpendicularmente ao sulco e centralizadas neste, sendo cinco de cada lado do meio do sulco (Figura 1). Entretanto, cada grupo de 10 amostras simples (10 prismas) foi coletado em um local escolhido de forma aleatória na área de plantio, sendo cada local distanciado de aproximadamente $10 \mathrm{~m}$ um do outro. Dessa forma, foram obtidas 30 amostras simples (30 prismas) para o volume de $360 \mathrm{~cm}^{3}, 30$ para o de $810 \mathrm{~cm}^{3}$ e 30 para o de $1.000 \mathrm{~cm}^{3}$. No PCDA, a amostragem foi realizada da mesma maneira, mas sem poder considerar a localização do sulco, uma vez que nesse sistema as amostras foram coletadas depois da aração-gradagens. As amostras simples foram secas ao ar, peneiradas ( $2 \mathrm{~mm})$, homogeneizadas e analisadas separadamente, sendo determinados o $\mathrm{pH}$ em água $(1: 2,5)$ e os teores de $\mathrm{P}$ e $\mathrm{K}$ disponíveis (Mehlich-1) e de $\mathrm{Ca}^{2+} \mathrm{e} \mathrm{Mg}^{2+}\left(\mathrm{KCl} 1 \mathrm{~mol} \mathrm{~L}^{-1}\right)$. 


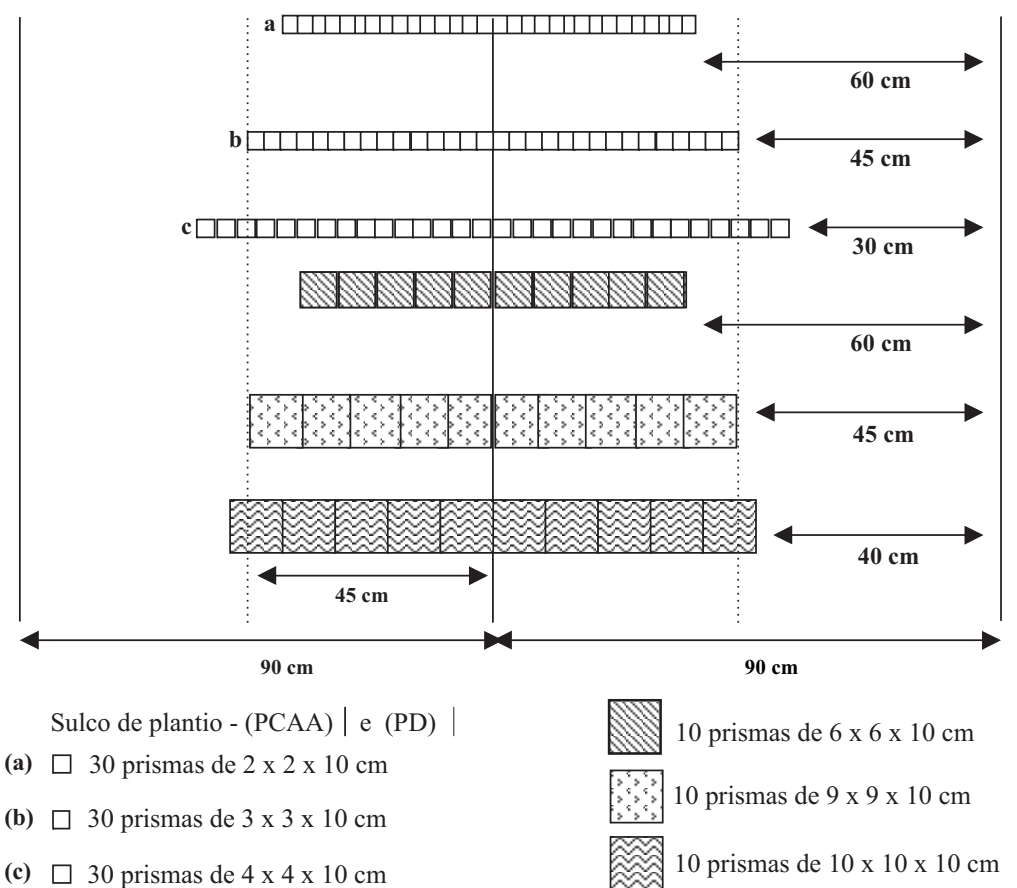

Figura 1. Esquema de coleta de amostras simples de solo com seis volumes diferentes $(40,90,160,360,810 \mathrm{e}$ $1.000 \mathrm{~cm}^{3}$ ), correspondendo, respectivamente, a prismas de base quadrada medindo $2,3,4,6,9 \mathrm{e} 10 \mathrm{~cm}$ de lado por $10 \mathrm{~cm}$ de profundidade, em duas situações de preparo-coleta (PD e PCAA). No PCDA, a amostragem foi realizada da mesma maneira, mas sem poder considerar a localização do sulco, devido à aração-gradagens do terreno.

O estudo foi analisado em um delineamento inteiramente casualizado, com três repetições (três amostras), formadas, cada uma, por 10 amostras simples de solo (10 prismas). A utilização desse artifício visava obter o erro das estimativas das variabilidades para cada volume estudado. Nos volumes de 40,90 e $160 \mathrm{~cm}^{3}$, uma amostra correspondeu às 10 amostras simples da parte média, e duas amostras corresponderam às 10 amostras simples externas de cada lado do sulco, uma vez que foram coletadas 30 amostras simples (30 prismas) seguidamente (Figura 1). Já para os volumes de 360, 810 e $1.000 \mathrm{~cm}^{3}$, cada amostra correspondeu a 10 amostras simples (10 prismas) coletadas seguidamente (Figura 1), mas em três locais de amostragem escolhidos de forma aleatória em cada área de plantio.

Foram determinadas as médias das características químicas do solo avaliadas e as médias dos respectivos desvios-padrão e coeficientes de variação (três amostras). Foram considerados elevados os coeficientes de variação (CV) que proporcionaram o cálculo de um número de amostras simples superior a 20, utilizando-se a fórmula proposta por Cline (1944): $\mathrm{n}=\left(\mathrm{t}_{\alpha / 2} \mathrm{CV} / \mathrm{f}\right)^{2}$, com desvio em torno da média (f) de $10 \%$ e o valor tabelado da distribuição t de Student $\left(\mathrm{t}_{\alpha / 2}\right)$ igual a $2,262($ referente a $5 \%$ de probabilidade e $\mathrm{n}-1=9$ ). Dessa forma, coeficientes de variação acima de $20,26 \%$ foram considerados elevados no presente estudo.
Foi realizada análise de variância dos desviospadrão e dos coeficientes de variação por situação de preparo-coleta (Volumes, 5 graus de liberdade; Resíduo, 12 graus de liberdade). Os desvios-padrão das características avaliadas nas três situações de preparo-coleta (PD, PCAA e PCDA) foram comparados pelo teste $t$ de Student. Como o teste $t$ compara a diferença de magnitude entre valores, quanto menor foi o seu resultado, em módulo, menor foi a diferença entre as estimativas das variabilidades das situações de preparo-coleta estudadas. Além disso, considerando determinada característica química do solo, a ausência de significância no teste $t$ representou, na média dos seis volumes, a mesma magnitude para estimativa da variabilidade nas situações de preparo-coleta testadas.

Utilizando análise de regressão, foram selecionados os modelos que melhor explicassem o comportamento das estimativas da variabilidade das características avaliadas, em função do volume das amostras simples de solo, com base no comportamento esperado (que, no caso, seria: redução na variabilidade até valores que praticamente se estabilizariam, em relação ao aumento do volume das amostras simples de solo), na significância dos coeficientes e no $\mathrm{R}^{2}$ (Alvarez V. \& Alvarez, 2003). A partir das equações selecionadas, foi gerada uma fórmula matemática que permitiu o cálculo do diâmetro de trado necessário para coleta de determinado número de amostras simples de solo, nas 
três situações de preparo-coleta estudadas (PD, PCAA e PCDA).

\section{RESULTADOS E DISCUSSÃO}

Cada volume utilizado na amostragem do solo (40, $90,160,360,810$ e $1.000 \mathrm{~cm}^{3}$ ) gerou médias distintas para todas as características avaliadas, porém sem apresentar aumento ou redução consistentes em relação à variação do volume das amostras simples (Quadro 1).

A partir de cada volume das amostras simples foram estimadas, também, diferentes variabilidades para as características avaliadas, medidas pelo desviopadrão (s) e pelo coeficiente de variação $(\mathrm{CV})$ (Quadro 2). Entretanto, o CV não caracterizou corretamente a variabilidade em alguns casos, uma vez que sofreu grande influência da média $(\mathrm{CV}=$ $100 \mathrm{~s} / \bar{y}$ ), como pode ser observado pelos resultados de $\mathrm{P}$ no plantio direto (Quadros 1 e 2). Nesse caso, a variabilidade medida pelo desvio-padrão foi menor com a amostra de $90 \mathrm{~cm}^{3}(13,33)$ do que com a de $160 \mathrm{~cm}^{3}$ $(19,93)$ e maior com a amostra de $810 \mathrm{~cm}^{3}(8,34)$ do que com a de $1.000 \mathrm{~cm}^{3}(5,19)$. Contudo, a média dos teores de $\mathrm{P}$ determinados nesses volumes não foi proporcional aos acréscimos e decréscimos no desviopadrão (Quadros 1 e 2). Assim, a variabilidade medida pelo CV foi maior com a amostra de $90 \mathrm{~cm}^{3}(39,40 \%)$ do que com a de $160 \mathrm{~cm}^{3}(27,11 \%)$ e menor com a amostra de $810 \mathrm{~cm}^{3}(29,42 \%)$ do que com a de $1.000 \mathrm{~cm}^{3}(31,21 \%)$, o que é o inverso da "real" variabilidade, medida pela variância $\left(\mathrm{s}^{2}\right): 177,69$ para $90 \mathrm{~cm}^{3} ; 397,20$ para $160 \mathrm{~cm}^{3} ; 69,56$ para $810 \mathrm{~cm}^{3}$; e 26,94 para $1.000 \mathrm{~cm}^{3}$.

Em termos médios, a variabilidade das características avaliadas, medida pelo coeficiente de variação, seguiu a ordem: $\mathrm{pH}<\mathrm{Ca}^{2+}<\mathrm{Mg}^{2+}<\mathrm{K}<\mathrm{P}$ (Quadro 2). Resultados semelhantes foram observados por diversos autores, tanto em plantio convencional (Barreto et al., 1974; Alvarez V. \& Carraro, 1976; Santos \& Vasconcellos, 1987; Saraiva et al., 1992) como em plantio direto (Salet et al., 1996; Anghinoni \& Salet, 1998; Schlindwein \& Anghinoni, 2000; Alvarez V. \& Guarçoni M., 2003). Deve-se ressaltar que, coletando-se 30 amostras simples (30 prismas) com volume de $90 \mathrm{~cm}^{3}$, todas retiradas dentro de uma distância de $90 \mathrm{~cm}$ (Figura 1), a variabilidade de $\mathrm{P}$ (CV) foi elevada, tanto no PD (36,93 \%) e no PCAA (31,21 \%) quanto no PCDA (20,40\%), demonstrando que a variabilidade é intensa e ocorre a curtas distâncias no solo, como observado por van den Hende \& Cottenie (1960), Jackson (1976), Alvarez V. \&

Quadro 1. Médias ${ }^{(1)}$ de características químicas da fertilidade, de acordo com o volume das amostras simples utilizado, em três situações de preparo-coleta

\begin{tabular}{|c|c|c|c|c|c|c|c|c|}
\hline \multirow{2}{*}{ Característica } & \multirow{2}{*}{ Situação ${ }^{(2)}$} & \multicolumn{7}{|c|}{ Volume da amostra simples $\left(\mathrm{cm}^{3}\right)$} \\
\hline & & 40 & 90 & 160 & 360 & 810 & 1.000 & $\bar{y}$ \\
\hline \multirow[t]{3}{*}{$P\left(\mathrm{mg} \mathrm{dm}^{-3}\right)$} & $\mathrm{PD}$ & 49,5 & 36,1 & 73,4 & 36,9 & 29,2 & 18,3 & 40,6 \\
\hline & PCAA & 32,7 & 40,4 & 45,9 & 32,5 & 31,7 & 34,0 & 36,2 \\
\hline & PCDA & 24,7 & 28,1 & 29,1 & 25,8 & 26,4 & 25,9 & 26,7 \\
\hline \multirow[t]{3}{*}{$\mathrm{K}\left(\mathrm{mg} \mathrm{dm}^{-3}\right)$} & $\mathrm{PD}$ & 261 & 308 & 378 & 302 & 311 & 276 & 306 \\
\hline & PCAA & 228 & 204 & 256 & 193 & 238 & 245 & 227 \\
\hline & PCDA & 234 & 229 & 243 & 203 & 184 & 171 & 211 \\
\hline \multirow[t]{3}{*}{$\mathrm{Ca}^{2+}\left(\mathrm{cmol}_{\mathrm{c}} \mathrm{dm}^{-3}\right)$} & $\mathrm{PD}$ & 6,45 & 6,65 & 6,75 & 6,09 & 5,77 & 6,05 & 6,29 \\
\hline & PCAA & 4,44 & 4,26 & 4,68 & 4,27 & 4,44 & 4,52 & 4,43 \\
\hline & PCDA & 2,65 & 2,57 & 2,73 & 2,93 & 3,05 & 2,72 & 2,78 \\
\hline \multirow[t]{3}{*}{$\mathrm{Mg}^{2+}\left(\mathrm{cmol}_{\mathrm{c}} \mathrm{dm}^{-3}\right)$} & $\mathrm{PD}$ & 1,86 & 1,63 & 1,93 & 1,17 & 1,37 & 1,23 & 1,53 \\
\hline & PCAA & 0,83 & 0,74 & 0,74 & 0,67 & 0,66 & 0,70 & 0,72 \\
\hline & PCDA & 0,55 & 0,53 & 0,62 & 0,63 & 0,55 & 0,69 & 0,60 \\
\hline \multirow[t]{3}{*}{$\mathrm{pH}$} & $\mathrm{PD}$ & 6,70 & 6,82 & 6,42 & 6,44 & 6,53 & 6,35 & 6,54 \\
\hline & PCAA & 5,34 & 5,38 & 5,31 & 5,40 & 5,32 & 5,32 & 5,35 \\
\hline & PCDA & 5,37 & 5,32 & 5,38 & 5,25 & 5,35 & 5,46 & 5,35 \\
\hline
\end{tabular}

(1) Média de três amostras (10 amostras simples cada) por volume $\left(\bar{y}_{\mathrm{ij}}\right) .{ }^{(2)} \mathrm{PD}$ : plantio direto; PCAA: plantio convencional antes da aração; PCDA: plantio convencional depois da aração. 
Quadro 2. Desvios-padrão ${ }^{(1)}$ e coeficientes de variação ${ }^{(2)}$ de características químicas da fertilidade, de acordo com o volume das amostras simples utilizado, em três situações de preparo-coleta

\begin{tabular}{|c|c|c|c|c|c|c|c|c|}
\hline \multirow{2}{*}{ Característica } & \multirow{2}{*}{ Situação ${ }^{(2)}$} & \multicolumn{7}{|c|}{ Volume da amostra simples $\left(\mathrm{cm}^{3}\right)$} \\
\hline & & 40 & 90 & 160 & 360 & 810 & 1.000 & $\bar{y}$ \\
\hline & \multicolumn{8}{|c|}{ Desvio-padrão } \\
\hline \multirow{3}{*}{$\begin{array}{l}\mathrm{P} \\
\left(\mathrm{mg} \mathrm{dm}^{-3}\right)\end{array}$} & PD & 9,32 & 13,33 & 19,93 & 12,27 & 8,34 & 5,19 & 11,40 \\
\hline & PCAA & 3,02 & 12,93 & 16,91 & 6,16 & 4,58 & 4,34 & 7,99 \\
\hline & PCDA & 3,82 & 5,73 & 4,63 & 3,72 & 2,50 & 2,38 & 3,80 \\
\hline $\mathrm{K}$ & $\mathrm{PD}$ & 31,73 & 36,49 & 38,03 & 57,39 & 43,02 & 43,53 & 41,70 \\
\hline \multirow[t]{2}{*}{$\left(\mathrm{mg} \mathrm{dm}^{-3}\right)$} & PCAA & 40,09 & 45,36 & 54,26 & 51,40 & 41,95 & 40,93 & 45,67 \\
\hline & PCDA & 53,65 & 38,91 & 34,57 & 29,99 & 21,91 & 18,25 & 32,88 \\
\hline \multirow{3}{*}{$\begin{array}{l}\mathrm{Ca}^{2+} \\
\left(\mathrm{cmol}_{\mathrm{c}} \mathrm{dm}^{-3}\right)\end{array}$} & $\mathrm{PD}$ & 0,28 & 0,31 & 0,75 & 0,39 & 0,35 & 0,34 & 0,40 \\
\hline & PCAA & 0,31 & 0,50 & 1,05 & 0,24 & 0,23 & 0,23 & 0,43 \\
\hline & PCDA & 0,24 & 0,30 & 0,35 & 0,34 & 0,26 & 0,24 & 0,29 \\
\hline \multirow{3}{*}{$\begin{array}{l}\mathrm{Mg}^{2+} \\
\left(\mathrm{cmol}_{\mathrm{c}} \mathrm{dm}^{-3}\right)\end{array}$} & $\mathrm{PD}$ & 0,07 & 0,07 & 0,29 & 0,11 & 0,12 & 0,12 & 0,13 \\
\hline & PCAA & 0,07 & 0,08 & 0,11 & 0,06 & 0,06 & 0,06 & 0,07 \\
\hline & PCDA & 0,10 & 0,12 & 0,11 & 0,11 & 0,09 & 0,09 & 0,10 \\
\hline \multirow[t]{4}{*}{$\mathrm{pH}$} & $\mathrm{PD}$ & 0,07 & 0,10 & 0,14 & 0,16 & 0,15 & 0,14 & 0,13 \\
\hline & PCAA & 0,11 & 0,18 & 0,32 & 0,31 & 0,27 & 0,27 & 0,24 \\
\hline & PCDA & 0,19 & 0,24 & 0,16 & 0,17 & 0,13 & 0,12 & 0,17 \\
\hline & \multicolumn{8}{|c|}{ Coeficiente de variação (\%) } \\
\hline \multirow[t]{3}{*}{$\mathrm{P}$} & PD & 18,33 & 36,93 & 27,11 & 31,56 & 29,42 & 31,21 & 29,09 \\
\hline & PCAA & 8,87 & 31,21 & 32,23 & 18,72 & 14,59 & 12,76 & 19,73 \\
\hline & PCDA & 15,34 & 20,40 & 15,89 & 14,20 & 9,32 & 9,15 & 14,05 \\
\hline \multirow[t]{3}{*}{$\mathrm{K}$} & $\mathrm{PD}$ & 11,72 & 12,15 & 9,98 & 18,38 & 13,94 & 15,68 & 13,64 \\
\hline & PCAA & 17,95 & 22,19 & 21,72 & 26,57 & 17,64 & 16,87 & 20,49 \\
\hline & PCDA & 23,03 & 16,91 & 14,13 & 14,16 & 11,54 & 10,58 & 15,06 \\
\hline \multirow[t]{3}{*}{$\mathrm{Ca}^{2+}$} & $\mathrm{PD}$ & 4,30 & 4,65 & 11,00 & 6,52 & 6,17 & 5,73 & 6,40 \\
\hline & PCAA & 7,08 & 11,59 & 20,69 & 5,55 & 5,24 & 5,14 & 9,22 \\
\hline & PCDA & 9,08 & 11,49 & 12,51 & 11,77 & 8,43 & 8,63 & 10,32 \\
\hline \multirow[t]{3}{*}{$\mathrm{Mg}^{2+}$} & $\mathrm{PD}$ & 3,87 & 4,51 & 14,46 & 10,13 & 8,91 & 9,65 & 8,59 \\
\hline & PCAA & 8,22 & 11,19 & 14,82 & 9,47 & 9,19 & 8,80 & 10,28 \\
\hline & PCDA & 17,38 & 23,11 & 17,90 & 17,99 & 15,44 & 12,87 & 17,45 \\
\hline \multirow[t]{3}{*}{$\mathrm{pH}$} & $\mathrm{PD}$ & 1,05 & 1,43 & 2,14 & 2,50 & 2,25 & 2,28 & 1,94 \\
\hline & PCAA & 2,08 & 3,36 & 6,07 & 5,81 & 5,16 & 5,10 & 4,60 \\
\hline & PCDA & 3,50 & 4,60 & 2,97 & 3,28 & 2,47 & 2,26 & 3,18 \\
\hline
\end{tabular}

(1) Média obtida entre os desvios-padrão das três amostras (10 amostras simples cada) por volume (s $\left.\mathrm{s}_{\mathrm{ij}}\right){ }^{(2)}$ Média obtida entre os coeficientes de variação das três amostras (10 amostras simples cada) por volume $\left(\mathrm{CV}_{\mathrm{ij}}\right)$. ${ }^{(3)}$ PD: plantio direto; PCAA: plantio convencional antes da aração; PCDA: plantio convencional depois da aração. 
Carraro (1976), Saraiva et al. (1992) e Raun et al. (1998). Segundo Schlindwein \& Anghinoni (2002), a variabilidade dos teores de $\mathrm{P}$, dentre as características químicas da fertilidade, é a mais influenciada pelas microvariações $(<5 \mathrm{~cm})$ do solo.

Maior variabilidade dos teores de $\mathrm{P}$ foi observada no sistema plantio direto (PD) e no plantio convencional antes da aração (PCAA), em relação ao plantio convencional depois da aração (PCDA), uma vez que o resultado do teste $t$ foi positivo e significativo nos dois casos (PD vs PCDA e PCAA vs PCDA) (Quadros 2 e 3). A aplicação de fertilizantes localizadamente no sulco de plantio cria duas populações distintas quanto aos teores de nutrientes: uma com elevada concentração e outra com concentração menor, especialmente de nutrientes com pouca mobilidade no solo (James \& Hurst, 1995). Além disso, o fato de as amostras simples terem sido coletadas transversalmente aos sulcos de plantio proporcionou a detecção dessas duas populações, aumentando a estimativa da variabilidade, como descrito por Kitchen et al. (1990). No PCDA, ocorreu o revolvimento do solo pela aração, diminuindo a variabilidade dos teores de $\mathrm{P}$, como observado por Vasconcellos et al. (1982) e Santos \& Vasconcellos (1987).

A variabilidade dos teores de $P$, por outro lado, não apresentou diferença entre o PD e o PCAA (Quadros 2 e 3 - teste t não-significativo), visto que os sulcos estavam presentes no momento da amostragem do solo nos dois casos.

No caso do K, poder-se-ia esperar comportamento semelhante ao do $\mathrm{P}$, ou seja, maior variabilidade no PD em relação ao PCDA, uma vez que ambos os nutrientes foram aplicados localizadamente no sulco de plantio. Isso, entretanto, não ocorreu, sendo as variabilidades dos teores de K no PD e no PCDA muito semelhantes, como pode ser observado pelos valores médios (Quadros 2) e pela ausência de significância do teste t (Quadro 3). Entretanto, a variabilidade de
K não deve ser explicada unicamente pela aplicação no sulco. Klepker \& Anghinoni (1995) relatam alta concentração de K disponível na base dos colmos de plantas de milho, com diminuição gradativa a partir destes, em virtude da lavagem do K presente na parte área das plantas pela água da chuva e, ou, da exsudação pelas raízes. Para esses autores, a estratificação de $\mathrm{K}$ na superfície do solo, nos sistemas de cultivo com pequena mobilização, ocorre em razão da grande quantidade absorvida pelas plantas e do posterior acúmulo na superfície do solo pela lavagem da parte aérea, além das adubações. Concluem, ainda, que a distribuição de K no solo é mais influenciada pela lavagem da parte aérea do que pelo modo de aplicação do fertilizante, em qualquer método de preparo.

Para $\mathrm{pH}$, a maior variabilidade no PCAA e no PCDA em relação ao $\mathrm{PD}$, uma vez que os resultados do teste $t$ foram negativos e significativos para esses casos (PD vs PCAA e PD vs PCDA) (Quadros 2 e 3), pode ser explicada como efeito direto da aração. No PD, a aplicação de calcário é superficial e homogênea, o que proporciona menor estimativa da variabilidade para os valores de $\mathrm{pH}$. Ao se realizar a aração, pode ocorrer bandeamento do solo que foi corrigido, além de exposição de camadas com valores de $\mathrm{pH}$ mais baixos, resultando em maior variabilidade dos valores de $\mathrm{pH}$. Santos \& Vasconcellos (1987) mostraram que a variabilidade dos valores de $\mathrm{pH}$ no solo pode aumentar após a aração, sendo sua distribuição mais homogênea antes do processo de preparo.

A estimativa da variabilidade para os teores de $\mathrm{Ca}^{2+}$, por outro lado, diminuiu com o preparo do solo (PD vs PCDA, positivo e significativo pelo teste $\mathrm{t}$ ) (Quadros 2 e 3). Esse resultado pode parecer contraditório em relação ao obtido para os valores de $\mathrm{pH}$, uma vez que a fonte primária de Ca é o calcário. Contudo, o adubo fosfatado aplicado no sulco de plantio contém elevada concentração de $\mathrm{Ca}$, o que explicaria a menor variabilidade após o preparo do solo. A

Quadro 3. Valores de t de Student calculados para as comparações entre os desvios-padrão médios ${ }^{(1)}$ de características químicas da fertilidade do solo, em três situações de preparo-coleta

\begin{tabular}{|c|c|c|c|}
\hline \multirow{2}{*}{ Característica } & \multicolumn{3}{|c|}{ Situação ${ }^{(2)}$} \\
\hline & PD vs PCAA & PD vs PCDA & PCAA vs PCDA \\
\hline $\mathrm{P}$ & $1,382^{\mathrm{ns}}$ & $5,215^{* *}$ & $2,032^{0}$ \\
\hline $\mathrm{K}$ & $-0,634^{\mathrm{ns}}$ & $1,397^{\mathrm{ns}}$ & $2,852^{*}$ \\
\hline $\mathrm{Ca}^{2+}$ & $-0,176^{\mathrm{ns}}$ & $1,877^{0}$ & $1,061^{\mathrm{ns}}$ \\
\hline $\mathrm{Mg}^{2+}$ & $1,935^{\circ}$ & $0,940^{\mathrm{ns}}$ & $-2,821^{*}$ \\
\hline $\mathrm{pH}$ & $-5,819^{* *}$ & $-2,653^{*}$ & $3,510^{* *}$ \\
\hline
\end{tabular}

\footnotetext{
${ }^{(1)}$ Média dos seis volumes das amostras simples por situação de preparo-coleta $\left(\bar{y}_{\mathrm{i}}\right) .{ }^{(2)}$ PD: plantio direto; PCAA: plantio convencional antes da aração; PCDA: plantio convencional depois da aração; ${ }^{\text {ns: }}$ não-significativo; ${ }^{\text {o, }}$ " e ${ }^{* *}$ : significativo a 10,5 e $1 \%$, respectivamente.
} 
estimativa da variabilidade dos teores de $\mathrm{Mg}^{2+}$ não apresentou consistência de aumento ou redução em relação às situações de preparo-coleta (Quadros 2 e 3).

A partir dos dados obtidos, foi possível selecionar modelos que descrevessem a variabilidade em função do volume das amostras simples de solo, para todas as características avaliadas (Quadros 4 e 5).

A forma como se procedeu à amostragem do solo (Figura 1) teve grande influência nos resultados obtidos e, conseqüentemente, na escolha dos modelos. $\mathrm{O}$ conjunto de amostras simples (prismas) com volume de $160 \mathrm{~cm}^{3}$ foi o que teve maior influência do sulco de plantio (Figura 1), apresentando maior variabilidade, medida pelo desvio-padrão, na maioria dos casos, tanto no plantio direto $(\mathrm{PD})$ quanto no convencional antes da aração (PCAA) (Quadro 2).

Com exceção do $\mathrm{pH}$, as demais características apresentaram, em geral, aumento da variabilidade até a amostra simples de $160 \mathrm{~cm}^{3}$, diminuindo gradativamente, a partir desse volume, no PD e no PCAA (Quadro 2). Foi selecionado o modelo descontínuo linear-hiperbólico para essa situação (Quadros 4 e 5).

O comportamento de aumento e posterior redução na variabilidade das características avaliadas, em função da elevação do volume das amostras simples

Quadro 4. Equações de regressão dos desvios-padrão $(\hat{s})^{(1)}$ de características químicas da fertilidade, de acordo com o volume das amostras simples utilizado (v), em três situações de preparo-coleta

\begin{tabular}{|c|c|c|c|c|}
\hline Característica $^{(2)}$ & Situação ${ }^{(3)}$ & \multicolumn{2}{|c|}{ Equação de regressão } & $\mathrm{R}^{2}$ \\
\hline \multirow[t]{3}{*}{$\begin{array}{l}\mathrm{P} \\
\left(\mathrm{mg} \mathrm{dm}^{-3}\right)\end{array}$} & $\mathrm{PD}$ & $\begin{array}{l}\hat{S}=5,615+0,0887^{* *} \mathrm{v} \\
\hat{S}=4,168+2579,21^{* *} 1 / \mathrm{v}\end{array}$ & $\begin{array}{l}\forall 40<\mathrm{v}<160 \mathrm{~cm}^{3} \\
\forall 160<\mathrm{v}<1.000 \mathrm{~cm}^{3}\end{array}$ & 0,98 \\
\hline & PCAA & $\begin{array}{l}\hat{S}=0,128+0,1120^{* *} \mathrm{v} \\
\hat{S}=1,162+2428,22^{* *} 1 / \mathrm{v}\end{array}$ & $\begin{array}{l}\forall 40<\mathrm{v}<160 \mathrm{~cm}^{3} \\
\forall 160<\mathrm{v}<1.000 \mathrm{~cm}^{3}\end{array}$ & 0,93 \\
\hline & PCDA & $\hat{S}=2,343+324,43^{* *} 1 / \mathrm{v}$ & $\forall 90<\mathrm{v}<1.000 \mathrm{~cm}^{3}$ & 0,94 \\
\hline $\mathrm{K}$ & $\mathrm{PD}$ & $\hat{S}=\bar{s}=41,70$ & & \\
\hline \multirow[t]{2}{*}{$\left(\mathrm{mg} \mathrm{dm}^{-3}\right)$} & PCAA & $\begin{array}{l}\hat{S}=35,104+0,1186^{\circ} \mathrm{v} \\
\hat{S}=40,128+2488,27^{\circ} 1 / \mathrm{v}\end{array}$ & $\begin{array}{l}\forall 40<\mathrm{v}<160 \mathrm{~cm}^{3} \\
\forall 160<\mathrm{v}<1.000 \mathrm{~cm}^{3}\end{array}$ & 0,89 \\
\hline & PCDA & $\hat{S}=22,432+1323,08^{* *} 1 / \mathrm{v}$ & $\forall 40<\mathrm{v}<1.000 \mathrm{~cm}^{3}$ & 0,91 \\
\hline \multirow[t]{3}{*}{$\begin{array}{l}\mathrm{Ca}^{2+} \\
\left(\mathrm{cmol}_{\mathrm{c}} \mathrm{dm}^{-3}\right)\end{array}$} & $\mathrm{PD}$ & $\begin{array}{l}\hat{S}=0,051+0,0041^{* *} \mathrm{v} \\
\hat{S}=0,233+80,34^{* *} 1 / \mathrm{v}\end{array}$ & $\begin{array}{l}\forall 40<\mathrm{v}<160 \mathrm{~cm}^{3} \\
\forall 160<\mathrm{v}<1.000 \mathrm{~cm}^{3}\end{array}$ & 0,91 \\
\hline & PCAA & $\begin{array}{l}\hat{S}=0,017+0,0063^{*} \mathrm{v} \\
\hat{S}=-0,012+160,42^{* * *} 1 / \mathrm{v}\end{array}$ & $\begin{array}{l}\forall 40<\mathrm{v}<160 \mathrm{~cm}^{3} \\
\forall 160<\mathrm{v}<1.000 \mathrm{~cm}^{3}\end{array}$ & 0,93 \\
\hline & PCDA & $\hat{S}=\bar{S}=0,29$ & & \\
\hline \multirow[t]{3}{*}{$\begin{array}{l}\mathrm{Mg}^{2+} \\
\left(\mathrm{cmol}_{\mathrm{c}} \mathrm{dm}^{-3}\right)\end{array}$} & $\mathrm{PD}$ & $\begin{array}{l}\hat{S}=-0,038+0,0019^{* *} \mathrm{v} \\
\hat{S}=0,067+33,11^{*} 1 / \mathrm{v}\end{array}$ & $\begin{array}{l}\forall 40<\mathrm{v}<160 \mathrm{~cm}^{3} \\
\forall 160<\mathrm{v}<1.000 \mathrm{~cm}^{3}\end{array}$ & 0,91 \\
\hline & PCAA & $\begin{array}{l}\hat{S}=0,054+0,0003^{*} \mathrm{v} \\
\hat{S}=0,048+9,04^{* *} 1 / \mathrm{v}\end{array}$ & $\begin{array}{l}\forall 40<\mathrm{v}<160 \mathrm{~cm}^{3} \\
\forall 160<\mathrm{v}<1.000 \mathrm{~cm}^{3}\end{array}$ & 0,95 \\
\hline & PCDA & $\hat{S}=0,090+3,22^{0} 1 / \mathrm{v}$ & $\forall 90<\mathrm{v}<1.000 \mathrm{~cm}^{3}$ & 0,71 \\
\hline \multirow[t]{3}{*}{$\mathrm{pH}$} & $\mathrm{PD}$ & $\hat{S}=0,157-3,71^{* *} 1 / \mathrm{v}$ & $\forall 40<\mathrm{v}<1.000 \mathrm{~cm}^{3}$ & 0,90 \\
\hline & PCAA & $\hat{S}=0,316-8,42^{* *} 1 / \mathrm{v}$ & $\forall 40<\mathrm{v}<1.000 \mathrm{~cm}^{3}$ & 0,82 \\
\hline & PCDA & $\hat{S}=0,120+10,38^{* *} 1 / \mathrm{v}$ & $\forall 90<\mathrm{v}<1.000 \mathrm{~cm}^{3}$ & 0,86 \\
\hline
\end{tabular}


Quadro 5. Equações de regressão dos coeficientes de variação ( CV $)^{(1)}$ de características químicas da fertilidade, de acordo com o volume das amostras simples utilizado (v), em três situações de preparocoleta

\begin{tabular}{|c|c|c|c|c|}
\hline Característica $^{(2)}$ & Situação ${ }^{(3)}$ & \multicolumn{2}{|c|}{ Equação de regressão } & $\mathbf{R}^{2}$ \\
\hline \multirow[t]{3}{*}{$\mathrm{P}$} & $\mathrm{PD}$ & $\hat{C} V=\overline{C V}=29,09$ & & \\
\hline & PCAA & $\begin{array}{l}\hat{C} V=6,403+0,1831^{*} \mathrm{v} \\
\hat{C} V=9,373+3.623,08^{*} 1 / \mathrm{v}\end{array}$ & $\begin{array}{l}\forall 40 \leq \mathrm{v}<160 \mathrm{~cm}^{3} \\
\forall 160 \leq \mathrm{v} \leq 1.000 \mathrm{~cm}^{3}\end{array}$ & 0,83 \\
\hline & PCDA & $\hat{C} V=9,019+1.066,38^{* *} 1 / \mathrm{v}$ & $\forall 90 \leq \mathrm{v} \leq 1.000 \mathrm{~cm}^{3}$ & 0,92 \\
\hline \multirow[t]{3}{*}{$\mathrm{K}$} & $\mathrm{PD}$ & $\hat{C} V=\overline{C V}=13,64$ & & \\
\hline & PCAA & $\hat{C} V=\overline{C V}=20,49$ & & \\
\hline & PCDA & $\hat{C} V=11,281+478,35^{* *} 1 / \mathrm{v}$ & $\forall 40 \leq \mathrm{v} \leq 1.000 \mathrm{~cm}^{3}$ & 0,95 \\
\hline \multirow[t]{3}{*}{$\mathrm{Ca}^{2+}$} & $\mathrm{PD}$ & $\begin{array}{l}\hat{C} V=1,040+0,0580^{* *} \mathrm{v} \\
\hat{C} V=4,574+987,42^{*} 1 / \mathrm{v}\end{array}$ & $\begin{array}{l}\forall 40 \leq \mathrm{v}<160 \mathrm{~cm}^{3} \\
\forall 160 \leq \mathrm{v} \leq 1.000 \mathrm{~cm}^{3}\end{array}$ & 0,90 \\
\hline & PCAA & $\begin{array}{l}\hat{C} V=2,055+0,1145^{*} \mathrm{v} \\
\hat{C} V=0,637+3.025,92^{* *} 1 / \mathrm{v}\end{array}$ & $\begin{array}{l}\forall 40 \leq \mathrm{v}<160 \mathrm{~cm}^{3} \\
\forall 160 \leq \mathrm{v} \leq 1.000 \mathrm{~cm}^{3}\end{array}$ & 0,94 \\
\hline & PCDA & $\hat{C} V=\overline{C V}=10,32$ & & \\
\hline \multirow[t]{3}{*}{$\mathrm{Mg}^{2+}$} & PD & $\hat{C} V=\overline{C V}=8,59$ & & \\
\hline & PCAA & $\begin{array}{l}\hat{C} V=6,111+0,0548^{*} \mathrm{v} \\
\hat{C} V=7,362+1.139,55^{*} 1 / \mathrm{v}\end{array}$ & $\begin{array}{l}\forall 40 \leq \mathrm{v}<160 \mathrm{~cm}^{3} \\
\forall 160 \leq \mathrm{v} \leq 1.000 \mathrm{~cm}^{3}\end{array}$ & 0,97 \\
\hline & PCDA & $\hat{C} V=13,772+824,00^{* *} 1 / \mathrm{v}$ & $\forall 90 \leq \mathrm{v} \leq 1.000 \mathrm{~cm}^{3}$ & 0,86 \\
\hline \multirow[t]{3}{*}{$\mathrm{pH}$} & PD & $\hat{C} V=2,442-60,20^{* *} 1 / \mathrm{v}$ & $\forall 40 \leq \mathrm{v} \leq 1.000 \mathrm{~cm}^{3}$ & 0,88 \\
\hline & PCAA & $\hat{C} V=5,978-157,44^{* *} 1 / \mathrm{v}$ & $\forall 40 \leq \mathrm{v} \leq 1.000 \mathrm{~cm}^{3}$ & 0,82 \\
\hline & $\mathrm{PCDA}$ & $\hat{C} V=2,232+197,79^{* *} 1 / \mathrm{v}$ & $\forall 40 \leq \mathrm{v} \leq 1.000 \mathrm{~cm}^{3}$ & 0,84 \\
\hline
\end{tabular}

(1) Média obtida entre os coeficientes de variação das três amostras (10 amostras simples cada) por volume $\left(\mathrm{CV}_{\mathrm{ij}}\right) ; \hat{C} V: \mathrm{CV}$ estimado; $\overline{C V}$ : CV médio. ${ }^{(2)} \mathrm{PD}$ : plantio direto; PCAA: plantio convencional antes da aração; PCDA: plantio convencional depois da aração; * ${ }^{* *}$ : significativo a 5 e $1 \%$, respectivamente; $\forall$ : condicional, qualquer que seja o valor entre...

de solo, no PD e no PCAA (Quadros 4 e 5), pode ser explicado pela influência do sulco de plantio, o qual, segundo Mallarino (1996), causa dependência espacial com alcance próximo ao espaçamento entre sulcos.

Para $\mathrm{P}$ no plantio direto (PD), a causa dessa influência é clara, uma vez que o sulco foi amostrado com o conjunto de amostras simples de $160 \mathrm{~cm}^{3}$ (Figura 1). $\mathrm{O}$ fato de a variabilidade de $\mathrm{P}$ diminuir a partir da amostra de $160 \mathrm{~cm}^{3}$ deve-se ao aumento gradativo do volume das amostras simples, que incorpora no volume de solo coletado as variações horizontais a curtas distâncias, ou seja, as microvariações $(<5 \mathrm{~cm})$, diminuindo, conseqüentemente, a estimativa da variabilidade. Por isso, o conjunto de amostras com $1.000 \mathrm{~cm}^{3}$ apresentou menor estimativa da variabilidade dos teores de $\mathrm{P}$ do que o conjunto de $160 \mathrm{~cm}^{3}$ (Quadros $2 \mathrm{e}$ 4), apesar de também amostrar o sulco (Figura 1).
Nesse caso, o elevado volume da amostra de $1.000 \mathrm{~cm}^{3}$ diluiu a concentração de $\mathrm{P}$ do sulco, resultando em menor teor médio (Quadro 1) e menor variação entre as amostras simples (Quadro 2).

Para P no plantio convencional antes da aração (PCAA), pode ter ocorrido influência de sulcos de plantio da safra anterior, pois, segundo Vasconcellos et al. (1982), os teores de $\mathrm{P}$ em determinada área podem ser influenciados pelas adubações no sulco de plantio mesmo após a aração e gradagem, ou seja, em alguns pontos o adubo permanece no próprio sulco, ou distribuído ao acaso, nas entrelinhas de plantio. Dessa forma, o conjunto de amostras simples de $160 \mathrm{~cm}^{3}$ pode ter sido mais influenciado por possíveis dependências espaciais do que os demais conjuntos de amostras, pois o conjunto de amostras simples coletadas com $160 \mathrm{~cm}^{3}$ cobriu maior área do terreno (Figura 1). 
Para K no PCAA, haveria dependência espacial, diminuindo a partir do sulco, causada pela lavagem da parte aérea, como sugerido por Silva \& Ritchey (1982), a qual seria melhor detectada pelo conjunto de amostras simples de $160 \mathrm{~cm}^{3}$ (Figura 1), causando, além de maior teor médio (Quadro 1), maior variabilidade dos teores de K neste volume (Quadros 2, 4 e 5).

Como $\mathrm{Ca}$ e $\mathrm{Mg}$ foram aplicados a lanço na forma de corretivo, não haveria, aparentemente, nenhuma influência do sulco de plantio. Todavia, o conjunto de amostras simples de $160 \mathrm{~cm}^{3}$ proporcionou maiores teores de $\mathrm{Ca}^{2+}$ no PD e no PCAA e de $\mathrm{Mg}^{2+}$ no PD (Quadro 1). Além disso, ocorreu aumento da

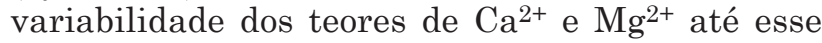
volume, com posterior redução em relação à elevação do volume das amostras simples, tanto no PD quanto no PCAA (Quadros 2, 4 e 5). Assim, pode-se inferir que os teores e a variabilidade de $\mathrm{Ca}^{2+} \mathrm{e} \mathrm{Mg}^{2+}$ tenham sido influenciados pela localização do sulco de plantio. Isso ocorreria devido ao Ca contido no fertilizante fosfatado e a um intenso transporte por fluxo em massa, causando maior concentração desses nutrientes na região próxima às raízes.

A variabilidade de $\mathrm{pH}$ no $\mathrm{PD}$ e no PCAA apresentou comportamento completamente inverso ao esperado, com elevação da estimativa da variabilidade à medida que se aumentou o volume das amostras simples, até um patamar em que esta estimativa se manteve praticamente estável (Quadros 4 e 5). Isso pode ser explicado por uma possível dependência espacial dos valores de $\mathrm{pH}$ a distâncias menores do que as microvariações do solo $(<5 \mathrm{~cm})$. Esse fato foi comprovado por Yang et al. (2001), que determinaram valores de $\mathrm{pH}$ a cada centímetro de amostras indeformadas de solo medindo 1,0 × 2,5 cm de lado e $10 \mathrm{~cm}$ de espessura e observaram dependência espacial dos valores de $\mathrm{pH}$, medida por meio de semivariograma, com alcance variando de 3,5 a $4,5 \mathrm{~cm}$.

No plantio convencional depois da aração (PCDA), para todas as características químicas da fertilidade, com exceção do teor de $\mathrm{Ca}^{2+}$, para o qual não foi possível obter um modelo, foi selecionado apenas o modelo hiperbólico para todo o espaço amostral, ou seja, houve redução nos valores das estimativas da variabilidade até que praticamente se estabilizaram, em função do aumento do volume das amostras simples de solo (Quadros 4 e 5). Nesse caso, não havia influência ou dependência espacial causada pelos sulcos de plantio. Assim, a distribuição dos teores no solo ocorreu de forma aleatória, gerando redução na variabilidade em função do aumento gradativo do volume das amostras simples de solo. Schlindwein \& Anghinoni (2002) também observaram redução na variabilidade dos teores de $\mathrm{Pe} \mathrm{K}$ com o aumento do volume das amostras simples de solo coletadas de forma aleatória, porém em áreas sob plantio direto com adubação realizada sempre a lanço.
A partir do exposto, pode-se assumir que, caso a amostragem fosse realizada de forma aleatória, a distâncias maiores do que o espaçamento entre sulcos, o comportamento, não a magnitude (valor), da estimativa da variabilidade de todas as características avaliadas seria o mesmo no PD, no PCAA e no PCDA, ou seja, redução na variabilidade em função do aumento gradativo do volume das amostras simples de solo, uma vez que seria ultrapassado o alcance da dependência espacial gerada pelos sulcos de plantio. Isso não quer dizer que as áreas como um todo não pudessem apresentar dependência espacial causada pelas macrovariações $(>2 \mathrm{~m})$, a qual poderia ser avaliada apenas por amostragens de solo adequadas aos estudos geoestatísticos.

Como as amostras simples de solo (prismas) foram coletadas numa pequena área de cada um dos três talhões homogêneos utilizados para amostragem (PD, PCAA e PCDA), poder-se-ia supor pequena representatividade da amostragem, considerando toda a área dos talhões. Por outro lado, a coleta de amostras simples contemplou as duas populações distintas quanto aos teores de nutrientes descritas por James \& Hurst (1995), uma com elevada concentração (sulco) e outra com concentração menor (entrelinha).

Essa fonte de variação (sulco-entrelinha), por sua vez, é a principal responsável pela elevada variabilidade obtida na amostragem do solo em áreas nas quais sejam mantidos os sulcos de plantio, caso do PD e do PCAA. Exatamente por isso, Guarçoni M. et al. (2006), coletando amostras em plantio direto e plantio convencional antes da aração, observaram que a estimativa da variabilidade dos teores de $\mathrm{P}$ de uma pequena área de $1,228 \mathrm{~m}^{2}$ foi, em cada um dos casos, muito semelhante à variabilidade de todo o talhão homogêneo, sendo esta estimada a partir de 275 amostras simples coletadas aleatoriamente. Dessa forma, nada impede que o cálculo do número de amostras simples, necessário para formar uma amostra composta representativa, seja realizado a partir das estimativas das variabilidades dos teores de $\mathrm{P}$ observadas no presente estudo, podendo ser extrapolado para cada um dos três talhões homogêneos estudados (PD, PCAA e PCDA).

Como o CV pode sofrer influência direta da média estimada para a amostra em questão (formada por 10 amostras simples) e, conseqüentemente, mascarar a variabilidade medida pelo desvio-padrão (s), optou-se por estimar equações de regressão para a característica que apresentou maior variabilidade (P) (Quadro 2), com base no desvio-padrão expresso em \% da média geral (CVM), por situação de preparo-coleta (Quadro 6). Deve-se notar que os valores de $\mathrm{R}^{2}$, nesse caso (Quadro 6), são os mesmos apresentados pelas equações dos desvios-padrão (Quadro 4), como se deveria esperar.

Com base nos modelos hiperbólicos selecionados para os desvios-padrão dos teores de $\mathrm{P}$, expressos em \% da média geral (CVM) (Quadro 6), e supondo o modelo hiperbólico válido para todo o espaço amostral (40 a 
$1.000 \mathrm{~cm}^{3}$ ) (isso aconteceria se a amostragem fosse realizada de forma aleatória), foram calculados os diâmetros de trado necessários para coleta de definido número de amostras simples, em pequenas áreas ou em talhões homogêneos, no PD, no PCAA e no PCDA (Quadro 7). Essa recomendação pode ser considerada coerente, uma vez que na avaliação da fertilidade média do solo não há grande relação entre a estimativa da variabilidade de características químicas do solo e o tamanho da área a ser amostrada, bastando que esta seja considerada homogênea, como observado por Guarçoni M. et al. (2006).
Como a maioria dos trados utilizados na amostragem de solo apresenta diâmetro $\leq 4 \mathrm{~cm}$, o número de amostras simples geralmente preconizado por talhão homogêneo, no plantio convencional (20 a 30), só poderia ser utilizado para o PCDA (Quadro 7). Por isso, Vasconcellos et al. (1982) sugeriram que a amostragem de solo fosse realizada após aração e gradagem do terreno, visto que nesta situação a variabilidade dos teores de $\mathrm{P}$ seria reduzida, permitindo a utilização de trados com menor diâmetro para coleta de 20 a 30 amostras simples por talhão homogêneo.

Quadro 6. Equações de regressão para os desvios-padrão dos teores de P, expressos em \% da média geral $(\mathrm{CVM})^{(1)}$, em função do volume das amostras simples utilizado, em três situações de preparo-coleta

\begin{tabular}{cllll}
\hline Característica $^{(2)}$ & Situação $^{(3)}$ & Equação de regressão $^{2}$ & $\mathbf{R}^{2}$ \\
\hline $\mathrm{P}$ & $\mathrm{PD}$ & $\hat{C} V M=13,843+0,2188^{* *} \mathrm{v} \quad \forall 40 \leq \mathrm{v}<160 \mathrm{~cm}^{3}$ & \\
& & $\hat{C} V M=10,276+6.358,12^{* *} 1 / \mathrm{v}$ & $\forall 160 \leq \mathrm{v} \leq 1.000 \mathrm{~cm}^{3}$ & 0,98 \\
& PCAA & $\hat{C} V M=0,355+0,3093^{*} \mathrm{v}$ & $\forall 40 \leq \mathrm{v}<160 \mathrm{~cm}^{3}$ & 0,93 \\
& $\hat{C} V M=3,210+6.707,88^{* *} 1 / \mathrm{v} \quad \forall 160 \leq \mathrm{v} \leq 1.000 \mathrm{~cm}^{3}$ & 0,94 \\
\hline
\end{tabular}

(1) CVM: $100 \mathrm{~s}_{\mathrm{ij}} / \bar{y} \cdot{ }^{(2)} \mathrm{PD}$ : plantio direto; PCAA: plantio convencional antes da aração; PCDA: plantio convencional depois da aração; ${ }^{*} \mathrm{e}^{* *}$ : significativo a 5 e $1 \%$, respectivamente; $\forall$ : condicional, qualquer que seja o valor entre...

Quadro 7. Diâmetros de trado $(\varnothing)^{(1)}$ necessários para coleta de determinado número de amostras simples de solo, considerando os coeficientes de variação médios ( $\hat{C} V M)$ dos teores de $\mathbf{P}$ disponível obtidos a partir das equações de regressão (modelo hiperbólico), em três situações de preparo-coleta, com f = 10 e $20 \%$

\begin{tabular}{|c|c|c|c|c|c|c|c|}
\hline \multirow[b]{2}{*}{ Situação ${ }^{(2)}$} & \multirow[b]{2}{*}{$\mathbf{F}^{(3)}$} & \multicolumn{6}{|c|}{ Número de amostras simples } \\
\hline & & 5 & 10 & 15 & 20 & 30 & 40 \\
\hline & $\%$ & & 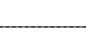 & 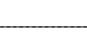 & & 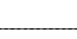 & 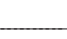 \\
\hline \multirow[t]{2}{*}{$\mathrm{PD}$} & 10 & - & 14,78 & 10,87 & 9,23 & 7,62 & 6,77 \\
\hline & 20 & 9,23 & 6,77 & 5,81 & 5,26 & 4,61 & 4,21 \\
\hline \multirow[t]{2}{*}{ PCAA } & 10 & 11,31 & 8,91 & 7,84 & 7,18 & 6,38 & 5,87 \\
\hline & 20 & 7,18 & 5,87 & 5,25 & 4,85 & 4,35 & 4,03 \\
\hline \multirow[t]{2}{*}{ PCDA } & 10 & 11,16 & 5,36 & 4,25 & 3,71 & 3,13 & 2,81 \\
\hline & 20 & 3,71 & 2,81 & 2,44 & 2,22 & 1,96 & 1,80 \\
\hline
\end{tabular}

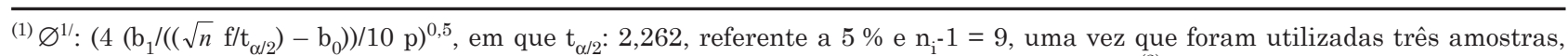
formadas por 10 amostras simples cada, na obtenção da equação para o CVM: f (v) (Quadro 6). ${ }^{(2)}$ PD: plantio direto; PCAA: plantio convencional antes da aração; PCDA: plantio convencional depois da aração. ${ }^{(3)}$ f: variação tolerada em torno da média.

${ }^{1 /}$ Partindo do modelo hiperbólico $\left(\mathrm{CVM}=\mathrm{b}_{0}+\mathrm{b}_{1} 1 / \mathrm{v}\right)$, tem-se: $\mathrm{v}=\mathrm{b}_{1} /\left(\mathrm{CVM}-\mathrm{b}_{0}\right)$; se $\mathrm{n}=\left(\mathrm{t}_{\alpha / 2} \mathrm{CVM} / \mathrm{f}\right) 2$, então: CVM $=\sqrt{n} \mathrm{f} / \mathrm{t}_{\alpha / 2} ;$ logo: $\mathrm{v}=\mathrm{b}_{1} /\left(\sqrt{n} \mathrm{f} / \mathrm{t}_{\alpha / 2}\right)-\mathrm{b}_{0}$ (Eq. 1); porém $\mathrm{v}=(\pi \varnothing 2 / 4) 10$ ou $\varnothing:(4 \mathrm{v} / 10 \pi) 0,5$ (Eq. 2). Substituindo Eq.1 na Eq. 2 , tem-se: $\varnothing:(4$ (b $1 /((\sqrt{n} \mathrm{f} /$ $\left.\left.\left.\left.\mathrm{t}_{\alpha / 2}\right)-\mathrm{b}_{0}\right)\right) / 10 \pi\right) 0,5$. 
Por outro lado, deve-se ressaltar que cada volume de amostra simples utilizado na amostragem prévia irá gerar um determinado número de amostras simples que fornecerá a mesma representatividade à amostra composta analisada. Isso é explicado por Burrough (1991), para quem o número de amostras simples é mais importante que sua localização ou seu volume, porém sem considerar o esforço e o tempo necessários para coleta de amostras de solo.

Nesse sentido, pequeno aumento no diâmetro do trado ou nas dimensões laterais da fatia de solo pode reduzir substancialmente o número de amostras simples necessário para formação de uma amostra composta representativa (Quadro 7), diminuindo, conseqüentemente, o esforço e o tempo de coleta. Assim, a realização da amostragem de solo após a aração, no intuito de diminuir o número de amostras simples necessário para caracterizar a área, sugerida por Vasconcellos et al. (1982), deixa de ter sentido, uma vez que se pode economizar uma aração para incorporação de corretivos apenas aumentando-se o diâmetro do trado.

Para coleta de 20 a 30 amostras simples utilizandose $\mathrm{f}=10 \%$, os diâmetros de trado calculados são elevados, tanto para o PD quanto para o PCAA (Quadro 7), tornando-se difícil encontrar no comércio trados com os diâmetros indicados. No entanto, considerando um desvio em torno da média de $20 \%$, o qual Schlindwein \& Anghinoni (2000) consideram mais adequado a condições de lavoura, os diâmetros calculados aproximam-se de possíveis diâmetros de trado encontrados no comércio, tanto para o PD quanto para o PCAA (Quadro 7).

Dessa forma, para as condições do presente estudo, pode ser recomendado, para pequenas áreas ou talhões homogêneos, um trado com diâmetro de aproximadamente $5,4 \mathrm{~cm}$, a ser utilizado para coleta de 20 amostras simples no PD ( $f=20 \%), 15$ no PCAA ( $f=20 \%$ ) e 10 ou cinco no PCDA (f = 10 e $20 \%$, respectivamente) (Quadro 7).

\section{CONCLUSÕES}

1. A estimativa da variabilidade da maioria das características químicas da fertilidade do solo avaliadas foi, de maneira geral, semelhante entre o plantio direto e o plantio convencional antes da aração, sendo maior em ambos do que no plantio convencional depois da aração.

2. O aumento do volume das amostras simples, para uma mesma profundidade de coleta, reduziu a estimativa da variabilidade das características químicas da fertilidade avaliadas até valores que praticamente se estabilizaram, permitindo a recomendação de um trado com diâmetro de $5,4 \mathrm{~cm}$, a ser utilizado para coleta de 20 amostras simples no plantio direto $(\mathrm{f}=20 \%), 15$ no plantio convencional antes da aração $(f=20 \%)$ e 10 no plantio convencional depois da aração $(f=10 \%)$.

\section{LITERATURA CITADA}

ALVAREZ V., V.H. \& ALVAREZ, G.A.M. Apresentação de equações de regressão e suas interpretações. B. Inf. SBCS, 28:28-32, 2003.

ALVAREZ V., V.H. \& CARRARO, I.M. Variabilidade do solo numa unidade de amostragem em solos de Cascavel e de Ponta Grossa, Paraná. R. Ceres, 23:503-510, 1976.

ALVAREZ V., V.H. \& GUARÇONI M., A. Variabilidade horizontal da fertilidade do solo de uma unidade de amostragem em sistema plantio direto. R. Bras. Ci. Solo, 27:297-310, 2003

ANGHINONI, I. \& SALET, R.L. Amostragem do solo e as recomendações de adubação e calagem no sistema plantio direto. In: NUERNBERG, N.J., ed. Conceitos e fundamentos do sistema plantio direto. Lages, Sociedade Brasileira de Ciência do Solo, 1998. p.27-52.

BARRETO, A.C.; NOVAIS, R.F. \& BRAGA, J.M. Determinação estatística do número de amostras simples de solo por área para avaliação da sua fertilidade. R. Ceres, 21:142147, 1974.

BURROUGH, P.A. Sampling designs for quantifying map unit composition. In: MUSBACH, M.J. \& WILDING, L.P., eds. Spatial variabilities of soil and landforms. Madison, Soil Science Society of America, 1991. p.89-125. (SSSA Special Publication, 28).

CLINE, M.G. Principles of soil sampling. Soil Sci., 58:275-288, 1944.

FANTE Jr., L.; REICHARDT, K.; JORGE, L.A.C. \& BACCHI, O.O.S. Distribuição do sistema radicular de uma cultura de aveia forrageira. Sci. Agric., 56:1091-1100, 1999.

GUARÇONI M., A.; ALVAREZ V., V.H.; NOVAIS, R.F.; CANTARUTTI, R.B.; LEITE, H.G. \& FREIRE, F.M. Definição da dimensão do indivíduo solo e determinação do número de amostras simples necessário à sua representação. R. Bras. Ci. Solo, 30:943-954, 2006.

JACKSON, M.L. Analisis quimico de suelos. Barcelona, Omega, 1976. 662p.

JAMES, D.W. \& HURST, R.L. Soil sampling technique for band-fertilized, no-till fields with Monte Carlo simulations. Soil Sci. Soc. Am. J., 59:1768-1772, 1995.

KITCHEN, N.R.; HAVLIN, J.L. \& WESTFALL, D.G. Soil sampling under no-till banded phosphorus. Soil Sci. Soc. Am. J., 54:1661-1665, 1990.

KLEPKER, D. \& ANGHINONI, I. Características físicas e químicas do solo afetadas por métodos de preparo e modos de adubação. R. Bras. Ci. Solo, 19:395-401, 1995.

MALLARINO, A.P. Spatial variability patterns of phosphorus and potassium in no-tilled for two sampling scales. Soil Sci. Soc. Am. J., 60:1473-1481, 1996. 
McBRATNEY, A.B. \& WEBSTER, R. How many observations are needed for regional estimation of soil properties? Soil Sci., 135:177-183, 1983

OLIVEIRA, F.H.T.; NOVAIS, R.F.; ALVAREZ V., V.H.; CANTARUTTI, R.B. \& BARROS, N.F. Fertilidade do solo no sistema plantio direto. In: ALVAREZ V., V.H.; SCHAEFER, C.E.G.R.; BARROS, N.F.; MELLO, J.W.V. \& COSTA, L.M., eds. Tópicos de ciência do solo. Viçosa, MG, Sociedade Brasileira de Ciência do Solo, 2002.v.2.p.393-486.

RAUN, W.R.; SOLIE, J.B.; JOHNSON, G.V.; STONE, M.L.; WHITNEY, R.W.; LEES, H.L.; SEMBIRING, H. \& PHILLIPS, S.B. Microvariability in soil test, plant nutrient, and yield parameters in bermudagrass. Soil Sci. Soc. Am. J., 62:683-690, 1998

SALET, R.L.; KRAY, C.H.; FORNARI, T.G.; CONTE, E.; KOCHHANN, R.A. \& ANGHINONI, I. Variabilidade horizontal e amostragem de solo no sistema plantio direto. In: REUNIÃO SUL-BRASILEIRA DE CIÊNCIA DO SOLO, 1., Lages, 1996. Resumos expandidos. Lages, Sociedade Brasileira de Ciência do Solo, 1996. p.74-76.

SANTOS, H.L. \& VASCONCELOS, C.A. Determinação do número de amostras de solo para análise química em diferentes condições de manejo. R. Bras. Ci. Solo, 11:97. $100,1987$.

SARAIVA, O.F.; ALVAREZ V., V.H. \& COSTA, L.M Variabilidade de algumas características físicas e químicas de um Podzólico Vermelho-Amarelo Câmbico distrófico. R. Ceres, 39:529-541, 1992.

SCHLINDWEIN, J.A. \& ANGHINONI, I. Tamanho da subamostra e representatividade da fertilidade do solo no sistema plantio direto. Ci. Rural, 32:963-968, 2002.
SCHLINDWEIN, J.A. \& ANGHINONI, I. Variabilidade horizontal de atributos de fertilidade e amostragem do solo no sistema plantio direto. R. Bras. Ci. Solo, 24:85-91, 2000 .

SILVA, J.E. \& RITCHEY, K.D. Acumulação diferencial de potássio em oxissolos devido à lavagem do nutriente das plantas de milho para o solo. R. Bras. Ci. Solo, 6:183-188, 1982.

SILVA, M.T.B. \& COSTA, E.C. Tamanho e número de unidades de amostra de solo para amostragem de larvas de Diloboderus abderus (Sturm) (Coleoptera: Melolonthidae) em plantio direto. An. Soc. Entomol., 27:193-197, 1998

UPCHURCH, D.R. \& EDMONDS, W.J. Statistical procedures for specific objectives. In: MUSBACH, M.J. \& WILDING, L.P., eds. Spatial variabilities of soil and landforms. Madison, Soil Science Society of America, 1991. p.49-71. (SSSA Special Publication, 28)

van den HENDE, A. \& COTTENIE, A. L'estimation de la fertilité du sol par les méthodes chimiques nouvelles. In: Comptes Rendus des Recherches; Travaux du Centre de Chemie Physique Agricole. Bruxelles, IRSIA, 1960. p.27174. (Bulletin, 25)

VASCONCELLOS, C.A.; SANTOS, H.L.; BAHIA FILHO, A.F.C.; OLIVEIRA, A.C. \& PACHECO, E.B. Amostragem de solo em área com adubação fosfatada aplicada a lanço e no sulco de plantio. R. Bras. Ci. Solo, 6:221-225, 1982.

WEBSTER, R. \& BURGESS, T.M. Sampling and bulking strategies for estimating soil properties in small regions. J. Soil Sci., 35:127-140, 1984.

YANG, J.J.; MOSBY, D.E.; CASTEEL, S.W. \& BLANCHAR, R.W. Microscale $\mathrm{pH}$ variability for assessing efficacy of phosphoric acid treatment in lead-contaminated soil. Soil Sci., 166:374-381, 2001. 